\title{
Analytical solutions for the profile of two-dimensional droplets with finite-length precursor films
}

\author{
Carlos Alberto Perazzo, ${ }^{1,2, *}$ J. R. Mac Intyre,,$^{3, \dagger}$ and J. M. Gomba ${ }^{3, \dagger}$ \\ ${ }^{1}$ IMeTTyB, Universidad Favaloro-CONICET, Solís 453, C1078AAI Buenos Aires, Argentina \\ ${ }^{2}$ Departamento de Física y Química, FICEN, Universidad Favaloro, Sarmiento 1853, C1044AAA Buenos Aires, Argentina \\ ${ }^{3}$ Instituto de Física Arroyo Seco IFAS (UNCPBA) and CIFICEN (UNCPBA-CICPBA-CONICET), Pinto 399, 7000, Tandil, Argentina
}

(Received 8 September 2016; revised manuscript received 31 October 2017; published 13 December 2017)

\begin{abstract}
By means of the lubrication approximation we obtain the full family of static bidimensional profiles of a liquid resting on a substrate under partial-wetting conditions imposed by a disjoining-conjoining pressure. We show that for a set of quite general disjoining-conjoining pressure potentials, the free surface can adopt only five nontrivial static patterns; in particular, we find solutions when the height goes to zero which describe satisfactorily the complete free surface for a finite amount of fluid deposited on a substrate. To test the extension of the applicability of our solutions, we compare them with those obtained when the lubrication approximations are not employed and under conditions where the lubrication hypothesis are not strictly valid, and also with axisymmetric solutions. For a given disjoining-conjoining potential, we report a new analytical solution that accounts for all the five possible solutions.
\end{abstract}

DOI: 10.1103/PhysRevE.96.063109

\section{INTRODUCTION}

The coverage of a solid surface with a liquid and the eventual emergence of stationary patterns are intensive areas of theoretical study due to its technological applications as much as for certain natural processes modeling. For example, we can mention the industrial coating processes $[1,2]$, the liquid lining of pulmonary alveoli and ocular surfaces by polymeric solutions $[3,4]$, and the pattern formation in dewetting of liquids $[5,6]$.

When liquid is deposited on a solid surface, the final morphology adopted by the fluid is the result of the competition among the different forces and effects involved in each problem such as surface tension, molecular interaction with the substrate, gravity, evaporation, condensation, centrifugation, chemical and/or physical inhomogeneities of the substrate and/or the liquid, stresses applied on the surface of the fluid, etc. (see Refs. [7-10]). Other relevant aspects are the amount of liquid and the extension of the substrate on which the liquid is deposited. It is possible to imagine that a given finite volume of liquid lying on a sufficiently large solid surface will not be able to totally cover it and the mass of fluid will take the shape of one (or various) droplet. But if the substrate is small, the liquid will reach its borders and then the contour conditions (dictated by the peculiarities of each situation) will be relevant to determine the shape of the surface of the liquid [11,12]. In conclusion, the surface of a liquid can adopt various different static shapes.

Many researchers have studied static configurations for a liquid layer on a substrate considering the surface tension, the molecular interaction between the liquid and the solid modeled by means of a disjoining pressure, and (sometimes) gravity. In his classical review [7], de Gennes derived the equation which gives the final spreading equilibrium. From this equation, he studied the structure of a drop near the contact line for organic

\footnotetext{
*perazzo@favaloro.edu.ar

†jmintyre@exa.unicen.edu.ar

łjgomba@exa.unicen.edu.ar
}

liquids (considering the effects of van der Waals forces) and for water solutions (with double layer effects). He also described a particular kind of flat droplet he called "pancake," for which he found an expression for its thickness and its edge (this type of drop was also discussed by Sharma [13]). In Ref. [14], Starov derived a slightly more general equation than that of Ref. [7], and discussed a solution describing a thin periodical liquid film and the values of its maximum and minimum were related to the characteristics of the disjoining pressure. Mitlin also analyzed periodical static configurations of liquid, but with the goal of establishing an analogy between dewetting and spinodal decomposition [15]. Bertozzi et al. [16] analyzed the existence and stability of a static liquid layer inside a bounded domain with particular border conditions, and they described a droplet whose leading order structure is a parabolic shape mounted over a thin uniform film with a thickness determined by the disjoining pressure. Starov and Velarde [17] described, for quite general disjoining pressure, a drop as having three regions: a central circular cap, a flat equilibrium film around the drop, and a transition zone. In Ref. [18], Thiele et al. included the effect of gravity and described some static solution for a liquid layer laterally unbounded. They showed a drop and a hole both mounted in an infinitely extended flat film, a kink profile that connects two infinitely extended flat films of different thicknesses, and a periodic profile, on which they concentrate their interest. Although many static profiles were described in the mentioned works, no systematic study linking the parameter space with the occurrence of each solution has been made.

Here we show the full bidimensional family of static profiles of a liquid resting on a substrate with which interacts by means of a disjoining-conjoining pressure. To do this we employ the lubrication or long wave approximation which is a successful and standard theoretical approach employed to study thin liquid films. The fundamental assumption is that the liquid surface has smooth variations so that the longitudinal scale of the liquid layer is much larger than its thickness. In contrast with previous works, we do not assume that the substrate is prewetted with an infinite precursor film of given thickness [5,19-21]. Nevertheless, we observe that for a certain range of 
parameters, a finite-length precursor film naturally appears as a part of the solution. Two parameters control the volume of the droplet and the length of this precursor film.

This article is organized as follows. In Sec. II the problem is stated and the governing equations are derived. In Sec. III we analyze the parameter space and discriminate five possible scenarios. We present the static solutions for each of the five parameter regions and also the analytical solution for a particular disjoining-conjoining pressure. This single expression accounts for all the five possible solutions. Section IV focuses on the solution that we consider the most interesting: a droplet surrounded by a finite-length precursor film (constant volume problem). In Sec. V we analyze how these static solutions are modified in the limit where the long wave approximation is not completely satisfied. Comparison with axisymmetric solutions are made in Sec. VI. Finally, in Sec. VII the conclusions and the final discussions are presented.

\section{PROBLEM STATEMENT}

Let us consider a flat substrate partially covered with a two-dimensional (2D) liquid layer of thickness $h \equiv h(x, t)$, viscosity $\mu$ and surface tension $\gamma, x$ and $z$ being the coordinates along and normal to the substrate, respectively, and $t$ the time. Under the lubrication hypothesis, the thickness of the profile must be much smaller than its extension so that it is possible to perform a perturbation series in powers of the small aspect ratio. Assuming that the Reynolds number satisfies $\operatorname{Re} \sim O(1)$, the continuity and the Navier-Stokes equations are simplified to a partial differential equation that describes the evolution of $h$ [8]:

$$
\begin{gathered}
\frac{\partial h}{\partial t}+\frac{\partial(h u)}{\partial x}=0 \\
u=\frac{h^{2}}{3 \mu} \frac{\partial}{\partial x}\left[\gamma \frac{\partial^{2} h}{\partial x^{2}}+\Pi(h)\right],
\end{gathered}
$$

where $u \equiv u(x, t)$ is the mean velocity (averaged in $z$ ) in the $x$ direction. The term with $\gamma$ models the capillarity and $\Pi$ is the disjoining-conjoining pressure, which takes into account the molecular interaction between the liquid and the substrate $[22,23]$. The model for $\Pi(h)$ that we employ is [7,24-28]

$$
\Pi(h)=\kappa\left[\left(\frac{h_{*}}{h}\right)^{n}-\left(\frac{h_{*}}{h}\right)^{m}\right], \quad n>m>1,
$$

which represents the competition between two antagonistic molecular forces. The parameter $\kappa$ is proportional to the Hamaker constant [29], and $h_{*}$ is the minimum of the potential $V$ defined as

$$
\begin{aligned}
V(h) & =-\int \Pi(h) d h \\
& =h_{*} \kappa\left[\frac{1}{n-1}\left(\frac{h_{*}}{h}\right)^{n-1}-\frac{1}{m-1}\left(\frac{h_{*}}{h}\right)^{m-1}\right] .
\end{aligned}
$$

We define the following dimensionless variables

$$
\hat{h}=\frac{h}{h_{*}}, \quad \hat{x}=\frac{x}{x_{c}}, \quad \hat{t}=\frac{\gamma h_{*}^{3}}{3 \mu x_{c}^{4}} t, \quad x_{c}^{2}=\frac{\gamma h_{*}}{\kappa},
$$

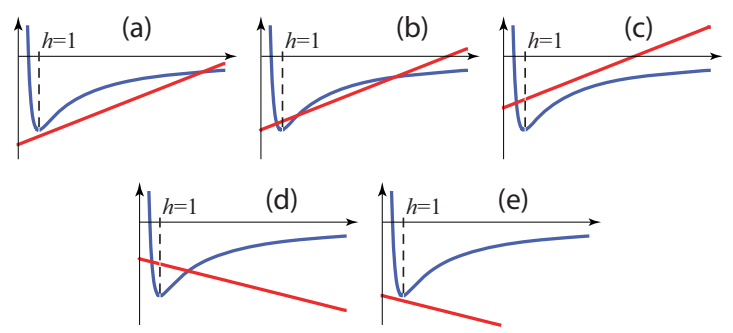

FIG. 1. Graphs of $v(h)$ (blue) and $P h-C$ (red) versus $h$. The five possible situations results in five qualitatively different solutions of Eq. (7) for any $n$ and $m$. The particular case between (a) and (b), where the straight line is tangent to $v(h)$, is analyzed as a limiting case in the text.

$$
\hat{u}=\frac{3 \mu x_{c}}{\kappa h_{*}^{2}} u, \quad \pi(h)=\frac{\Pi(h)}{\kappa}, \quad \text { and } \quad v(h)=\frac{V(h)}{h_{*} \kappa} .
$$

Replacing them in Eqs. (1) and (2) and omitting the hats for ease of reading, the first one remains unchanged and the second takes the form

$$
u=h^{2} \frac{\partial}{\partial x}\left[\frac{\partial^{2} h}{\partial x^{2}}+\pi(h)\right] .
$$

It is convenient to point out that to derive Eq. (6), it was assumed that $\frac{h_{*}}{x_{c}}|\partial h / \partial x| \ll 1$ so that the curvature was approximated by $\partial^{2} h / \partial x^{2}$.

\section{STATIC SOLUTIONS}

To look for static solutions of Eqs. (1) and (6) one has to require that $u=0$. Integrating twice, we get an equation that rules the shape of static profiles,

$$
h^{\prime}= \pm \sqrt{2} \sqrt{v(h)-P h+C},
$$

where the prime denotes derivation with respect to $x$, and $P$ and $C$ are integration constants. Equations equivalent to Eq. (7) were previously obtained in Refs. [7,18,30].

The shape of $v$ is independent of $n$ and $m$ and only quantitative details depend on these exponents: the potential diverges for $h=0$, decreases in the interval $(0,1)$, grows in $(1, \infty)$, has an inflection point at $h_{\text {inf }}=(n / m)^{1 /(n-m)}$, and tends to 0 for $h \rightarrow \infty$. To guarantee that the solution $h$ is real, the radicand in Eq. (7) must be nonnegative, i.e.,

$$
v(h) \geqslant P h-C \text {. }
$$

Therefore, from a graph of $v(h)$ and $P h-C$ versus $h$ it is possible to find out how many different static profiles exist and their general features.

Figure 1 shows all the possible scenarios. To analyze these graphs it is necessary to realize that: (1) there is solution only for those values of $h$ where the curve $v(h)$ is above of the straight line $P h-C$, (2) the points where they intersect each other are the values of $h$ where $h^{\prime}=0$, and (3) the points where the tangent straight line to the curve $v(h)$ is parallel to $P h-C$ are the inflection points (a similar analysis was done in Ref. [31] but restricted to only one of the solutions that will be shown here). Thus, in Fig. 1(a) the solution goes from $h=0$, where the slope diverges, to a finite $h>1$, where slope vanishes, given by the intersection of the curve and the 

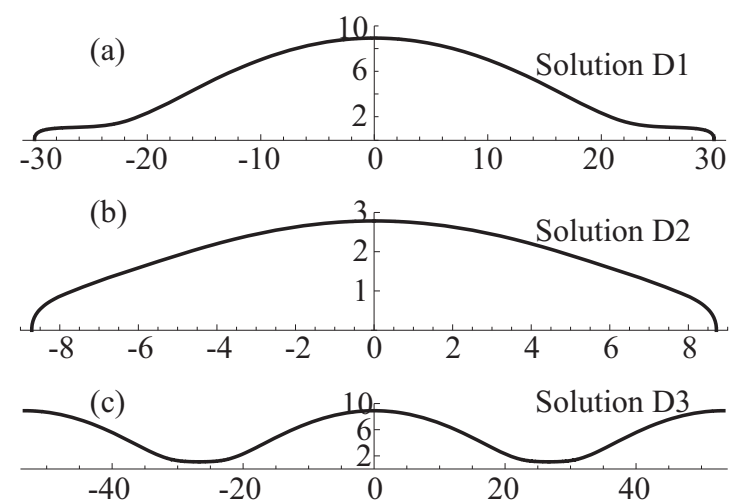

FIG. 2. Drop-shaped solutions. (a) Solution D1 for $P=0.05$ and $C=0.552$; (b) solution D2 for $P=0.16$ and $C=0.74$; (c) solution D3 for $P=0.05$ and $C=0.551 ; n=3$ and $m=2$ in the three cases.

straight line. If $P<P_{v, \max } \equiv(m / n)^{m /(n-m)}-(m / n)^{n /(n-m)}$ [the maximum slope of $v(h)$ ], the solution has inflection points with two different values of $h^{\prime}$ and its slope goes from infinity to zero but in a non-monotonous way. The profile is depicted in Fig. 2(a) and we shall denote this kind of solution as D1. On the contrary, if $P>P_{v \text {, max }}$, the solution is like the just described one but without inflection points and we shall call it D2, as shown in Fig. 2(b). The case shown in Fig. 1(b) allows two kinds of solutions. The first one is a D2 solution (here with $h \leqslant 1$ ). The second one, which we shall call D3, has a minimum and a maximum [where $h^{\prime}(x)=0$ ] and one inflection point between them. The D3 solution has a positive curvature at the minimum. Then, the profile is not allowed to decrease to zero and results in a periodic solution, as shown in Fig. 2(c). The last possibility for $P>0$ is shown in Fig. 1(c), where the solution is a D2 kind droplet but with $h<1$.

The last two scenarios correspond to $P<0$. In Fig. 1(d) there are two solutions, one is a D2 solution and the other, which we shall call ND1, is unbounded. The minimum occurs at $h>1$, it has no inflection point and the curvature is positive in the whole domain. Finally, in Fig. 1(e) there is one solution, which goes from $h=0$ to $h=\infty$, in both extremes $h^{\prime}=\infty$, and it has one inflection point. We will denote this kind of solution as ND2. Both ND1 and ND2 are presented in Fig. 3.

Varying the slope and the intercept of $P h-C$ one realizes that the situations just described cover all the possibilities and, as the shape of the potential $v(h)$ is independent of $n$ and $m$, this is true for any $n$ and $m$. Then, the conclusion is that for any $n$ and $m$ there are five nontrivial solutions of Eq. (7): D1, D2, D3, which have shape of droplets, and ND1 and ND2, which are nondroplet solutions. Below we summarize their main features:

(1) Solution D1 is a drop mounted over a finite-length precursor film of thickness $h_{f}>1$, as depicted in Fig. 2(a). It has a finite area and two inflection points in each half. The maximum height satisfies $h_{\max }>1$ and is the unique point where the slope of the profile is null. To the best of our knowledge, this solution has not been described in the literature, except in the limit case when the width of the precursor film is infinite, as, for example, in Ref. [18].
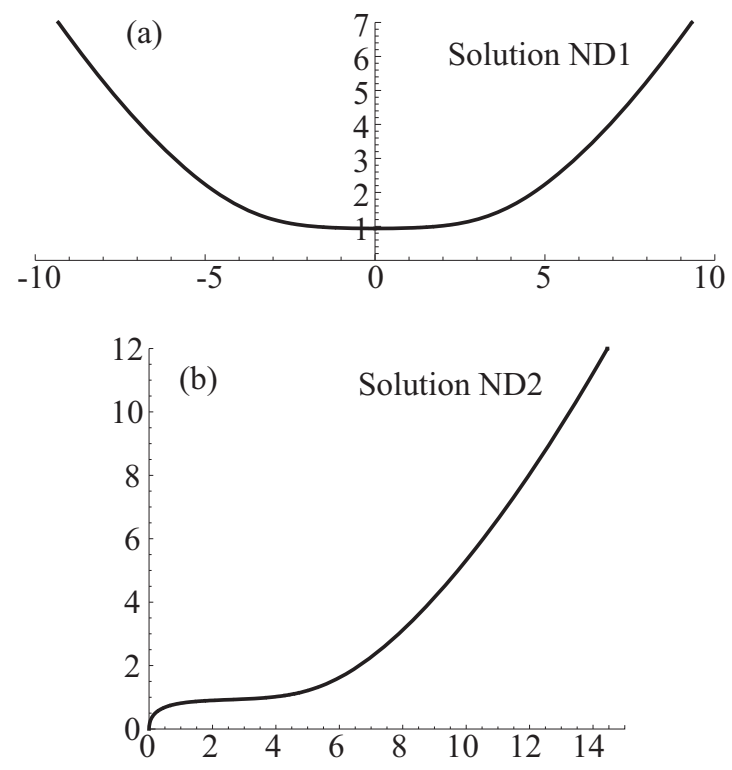

FIG. 3. Nondroplet solutions. (a) Solution ND1 with $P=-0.1$ and $C=0.404$; (b) solution ND2 with $P=-0.1$ and $C=0.405$; $n=3$ and $m=2$ in both cases.

(2) Solution D2 is a drop without precursor film, as shown in Fig. 2(b). This solution has a finite area, it does not have inflection points and its maximum height may be less than 1 . This solution may have different appearances: like the one in Fig. 2(b) with no limit for the height of the central parabolic cap, an small-area droplet with an almost half-circle profile, or may have a width much larger than its height with its central region almost horizontal as the "pancake" profile described in Refs. [7,13]. The transitions between these different shaped D2 solutions are smooth on $P$ and $C$.

(3) Solution D3 is an infinite succession of equally spaced identical drops mounted over a precursor film [see Fig. 2(c)]. Here $h>1$ and the solution has two inflections points (at the same height) in each cycle. It was previously studied extensively in Refs. [14,15,18].

(4) Solution ND1 is a symmetric divergent solution with a minimum [see Fig. 3(a)]. Its concavity is positive. When gravity is considered, this solution becomes the hole solution presented in Ref. [18].

(5) Solution ND2 is an asymmetric divergent solution with a precursor film [see Fig. 3(b)]. The solution has a single inflection point and the solution goes from $h=0$ to $h=\infty$. When the width of the precursor film diverges and gravity is considered, this solution becomes the kink solution presented in Ref. [18]. In Refs. [7,32], a similar solution is studied which describes an advancing contact line.

In Fig. 4 we show in a plane $(C, P)$ the regions where each solution is obtained. The limits of each region result from the analysis of Fig. 1. It can be deduced that the boundaries of these regions are the horizontal straight line $P=0$, the horizontal half-line given by $P=P_{v \text {,max }}$ (this value is the generalization of that given in Ref. [33] for $n=4$ and $m=3$ ) and $C>C_{v, \max } \equiv\left(m^{n-1} / n^{m-1}\right)^{1 /(n-m)}(n-m) /(n-1)(m-$ 


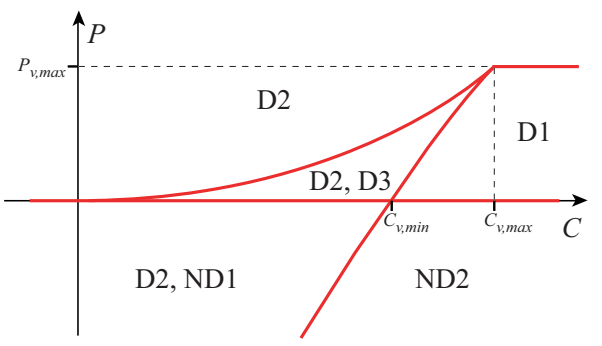

FIG. 4. Occurrence of each of the five solutions in the parameter space $P$ vs. $C$. The regions are delimited by the horizontal axes, the curve $\left(C_{v}, P_{v}\right)$ with $h_{f}>0$ [see Eq. (9)], and the half line with $P=P_{v, \max }$ and $C>C_{v, \max }$.

$1)$, and the curve $\left(C_{v}, P_{v}\right)$, with

$$
P_{v}=-\frac{1}{h_{f}^{n}}+\frac{1}{h_{f}^{m}}, \quad C_{v}=-\frac{n}{(n-1) h_{f}^{n-1}}+\frac{m}{(m-1) h_{f}^{m-1}},
$$

where $P_{v}$ and $-C_{v}$ are the slope and the ordinate of the tangent of $v(h)$, respectively, and $h_{f}>0$. Figure 4 allows us to see how the solutions change with $P$ and $C$. For example, starting from a solution D1, keeping $C$ fixed and decreasing $P$, the droplet will increase its area which will become infinity for $P=0$. For smaller values of $P$ the solution becomes of ND2 type, which can be seen as describing the precursor film of an infinitely large drop. On the other hand, if $P$ is kept constant and $C\left(\gtrsim C_{v}\right)$ is decreased, the precursor film becomes longer without any other change in the drop and it diverges when $C=C_{v}$. For smaller values of $C$, the solution becomes of the D3 kind with a decreasing period.

For the case $n=3$ and $m=2$, which corresponds to the competition of London van der Waals $(n=3)$ and ionicelectrostatics forces $(m=2)[29,34]$, Eq. (7) has an analytical implicit solution for any $P$ and $C$ which contains all the five solutions D1 to ND2:

$$
\begin{aligned}
x-x_{0} & =\sqrt{\frac{2}{P\left(h_{3}-h_{1}\right)}}\left(\left(h_{1}-h_{3}\right) \mathrm{E}(\psi, \tau)-h_{1} \mathrm{~F}(\psi, \tau)\right), \\
\psi & \equiv \arcsin \sqrt{\frac{h_{3}-h}{h_{3}-h_{2}}}, \quad \tau \equiv \frac{h_{3}-h_{2}}{h_{3}-h_{1}},
\end{aligned}
$$

where $h_{1}, h_{2}$, and $h_{3}$ are the roots of the polynomial $-2 P h^{3}+$ $2 C h^{2}-2 h+1$, and $\mathrm{F}(\psi, \tau)$ and $\mathrm{E}(\psi, \tau)$ are incomplete elliptic integrals of the first and second kind, respectively.

To the best of our knowledge, Eq. (7) cannot be solved analytically for general values of $n$ and $m$. In the cases $n=3$ and $m=2$ in Ref. [29], and $n=4$ and $m=3$ in Ref. [35] (corresponding to retarded and non retarded effects in Londonvan der Waals interactions), particular closed form solutions are presented which describe drops with a laterally unbounded precursor film (in Fig. 4 these solutions live in the left boundary of the region where D1 solutions live). Those solutions are only valid in the particular cases where $P=P_{v}$ and $C=C_{v}$, being $h_{f}$ the constant thickness of the precursor film with $1<h_{f}<(n / m)^{1 /(n-m)}$. Thus, Eq. (10) is a general solution of Eq. (7) that includes the particular case analyzed in Ref. [29].

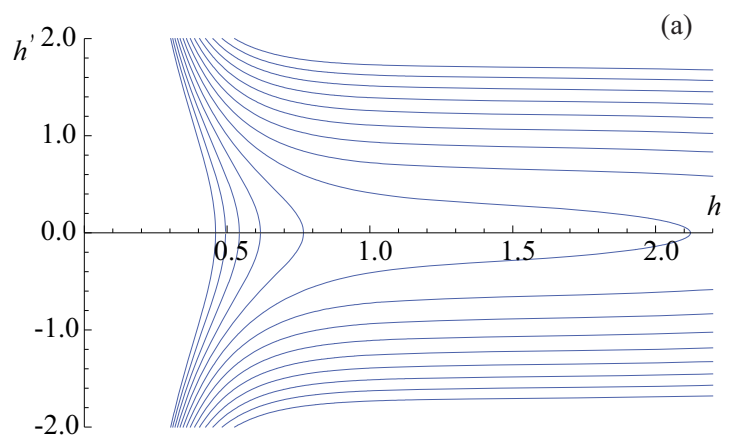

(a)

(b)

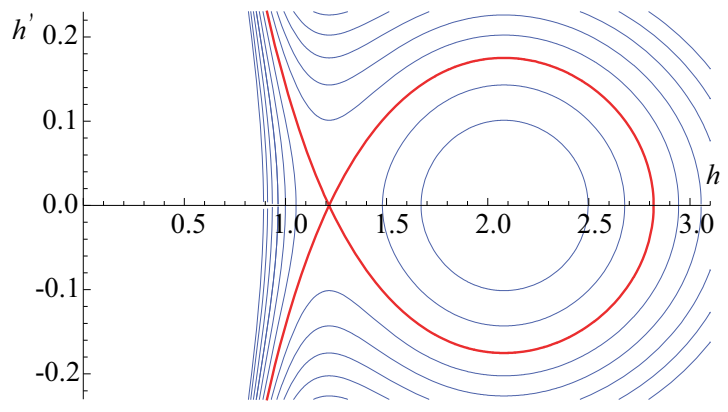

(c)

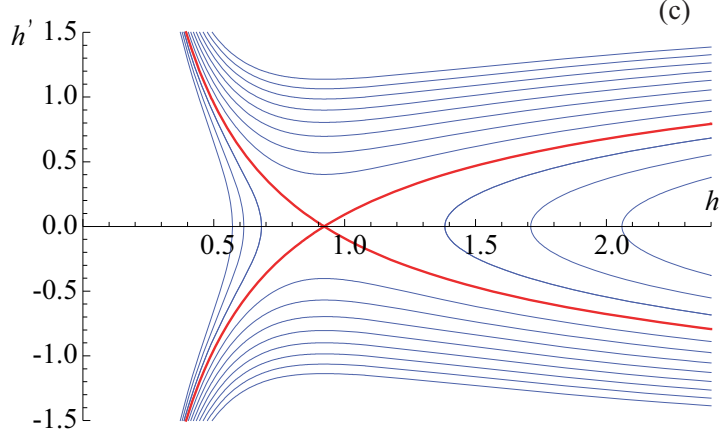

FIG. 5. Phase plane $h^{\prime}$ versus $h$ for fixed $P$ and different values of $C$. (a) $P=0.2$, (b) $P=0.12$, and (c) $P=-0.1$. In each panel the lines correspond to equally spaced values of $C$.

Analytical solutions for $P=0$ and $C=m /(m-1)-n /(n-$ 1) are given with $n=4$ and $m=3$ in Ref. [35] and with $n=5$ and $m=3$ in Ref. [36].

Notice that the slope of solutions D1, D2, and ND2 diverges when $h \rightarrow 0$, which is a gross violation of the assumptions of lubrication approximation. However, this does not mean that these solutions have to be discarded, it just imply that they are not reliable near $h=0$ (see Refs. [37,38] for a lengthy discussion about this point). The divergence of the slope when $h \rightarrow 0$ is due to the divergence of $v(h)$ in the same limit. Of course, this divergence of $v$ is non-physical and sometimes solved by introducing a cutoff (molecular) length that modifies the potential close to $h=0$, as we will do later in the text. The introduction of this cutoff only introduces small variations to the solution in the region close to the substrate.

The five stationary solution can also be analyzed in a phase plane spanned by $h$ and $h^{\prime}$, as it was done in Refs. [18,30]. In Fig. 5 we show three of such phase plane plots, each one with fixed $P$ and varying $C$. In the upper panel $P>P_{v \text {, max }}$ so that, according to Fig. 4, each curve correspond to a D2 solution. 
In Fig. 5(b), with $0<P<P_{v, \max }$, there are solutions D1, D2, and D3, depending of the value of $C$, which are separated by the thick red curve. Inside of the loop of this separatrix are located the D3 solutions, out of the loop and to the right are the D1 solutions, and to the left are the D2 solutions. In Fig. 5(c), with $P<0$, there are solutions D2 to the left, ND1 to the right, and ND2 over and below the separatrix. From this analysis can be confirmed that there is no other solution that those we describe here. Besides, the separatrix represents one of these five solution but in some limit (with $P=P_{v}$ and $C=C_{v}$ ), and its characteristics can be deduced from the kind of solutions that are at each side of it. For example, the loop of the thick red curve in the middle panel of Fig. 5 represents a D1 solution with an infinite precursor film, or equivalently, a D3 solution with infinite period, and the rest of the thick red curve represents two D2 solutions with a semi-infinite width (one with $h^{\prime}>0$, and the other with $h^{\prime}<0$ ). To the left of this separatrix, but very close to it, the D2 solution looks like a pancake-shaped droplet $[7,13]$ (this is also true for the Fig. 5(c)). The analytical expression for the solution given by the separatrix is given in Refs. [29,35] for $n=3, m=2$ and $n=4, m=3$, respectively. The phase planes shown in Fig. 5 are slightly different from those depicted in Refs. $[18,30]$, because in these works $\Pi$ is different from Eq. (3) and gravity was included. In Ref. [30] a periodic solution as D3 is extensively studied, but no other stationary solution was described. In Ref. [18], the authors describe three kinds of solutions: unbound solutions, similar to solutions ND1 and ND2; localized solutions, which are drops or depressions mounted over an infinite precursor film; and periodic solutions, which are analog to D3. However, they do not describe solutions with a finite lateral extension such as the solutions D1 and D2. Besides, we do not have "hole" or "kink" solutions (see Fig. 6 in Ref. [18]), because we do not include gravity in our problem.

The integration constants $P$ and $C$ may be chosen so that the solution has specific geometric characteristics. For example, if one looks for a solution with an inflection point at $h=h_{i}$ and a maximum (minimum) at $h=h_{m}>h_{i}\left(h_{m}<h_{i}\right)$, then $P=$ $-\pi\left(h_{i}\right)$ and $C=-v\left(h_{m}\right)-\pi\left(h_{i}\right) h_{m}$, or if the contact angle (defined as the angle of the interface at the upper inflection point, although other definition is possible, see Ref. [39]) has a predetermined value $\theta$, then $C=\frac{1}{2} \tan ^{2} \theta-v\left(h_{i}\right)-\pi\left(h_{i}\right) h_{i}$. On the other hand, from Eq. (7) it is possible to see the physical meaning of $P$ and $C$. If one derives this equation, it is obtained that $P=-h^{\prime \prime}-\pi(h)$, so that $P$ is the dimensionless excess pressure, which means that if $P$ is positive (negative) the pressure inside the liquid is larger (smaller) than the pressure in the ambient gas [34] (all these pressures were nondimensionalized by $\kappa$ ). Now, considering a macroscopic drop and evaluating Eq. (7) in the upper inflection point where its contact angle $\theta_{l}$ is measured, the radicand of Eq. (7) can be very well approximated by $C$ and this constant can be expressed as

$$
C=\frac{1}{2} \tan ^{2} \theta_{l}
$$

Furthermore, using the Young equation $\gamma_{s}=\gamma_{s l}+\gamma \cos \Theta_{l}$, where $\Theta_{l}$ is the contact angle for a large drop in dimension variables [from Eq. (5) $\tan \Theta_{l}=\frac{h_{*}}{x_{c}} \tan \theta_{l}$ ], Eq. (11)
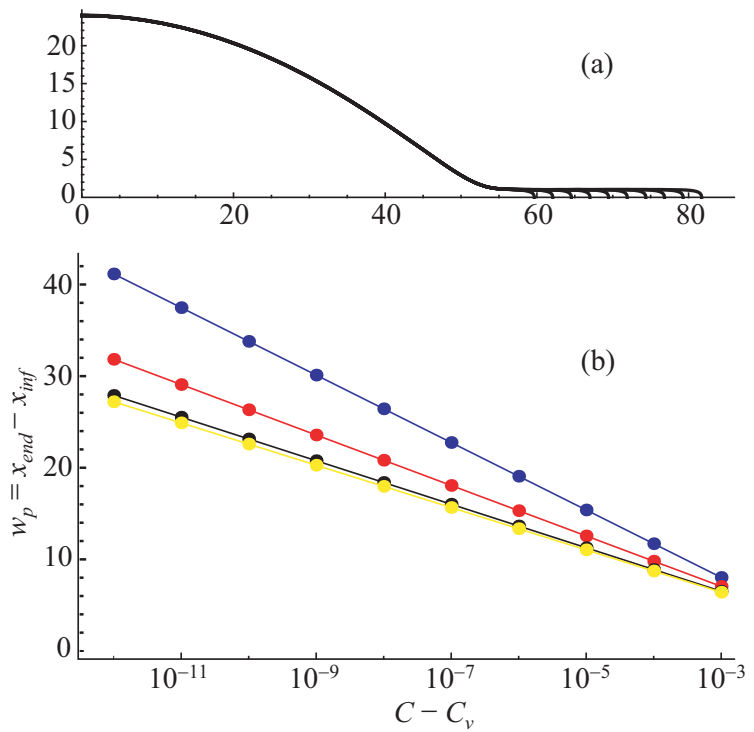

FIG. 6. Effect of $P$ and $C$ in the length $w_{p}$ of the precursor film. (a) $P=P_{v}$ is kept constant and $C-C_{v}=10^{-n}$ with $3 \leqslant n \leqslant 12$. Here $P_{v}=0.02$ and $C_{v}=0.520209$ is a point on the curve given by Eq. (9). The shape of the bulk is unchanged and only the precursor film increases its width. (b) The length $w_{p}$ of the precursor film measured as the difference between $x_{\text {end }}$ where $h=0$, and $x_{\text {inf }}$, the position with the largest curvature, i.e., where $h=(n / m)^{1 /(n-m)}$. The blue, red, black, and yellow points corresponds to $P=0.1,0.05,0.01$, and 0.001 , respectively. In this figure $(n, m)=(3,2)$.

leads to

$$
C=\frac{\gamma+\gamma_{s l}-\gamma_{s}}{h_{*} \kappa}=-\frac{S}{h_{*} \kappa},
$$

where $\gamma_{s l}$ and $\gamma_{s}$ are the interfacial tensions solid-liquid and solid-gas, respectively, and $S$ is the spreading coefficient. Then, $C$ is the dimensionless difference of energies between the substrate covered with a liquid layer [thick enough to disregard $v(h)]$ and the dry substrate. Therefore, $C$ must be positive for the case of partial wettability and $C=0$ for total wettability, so that more wettable is the solid, lesser is $C$, but always $C \geqslant 0$, and very importantly, given a pair solid substrate-liquid a unique value of $C$ is determined.

\section{THE SOLUTION D1}

The solution D1 is particularly attractive because it represents a droplet surrounded by a finite-length precursor film. The natural question is how this thickness profile depends on the parameters of the problem.

The height of the droplet, which is given by the unique real root of Eq. (7), decreases with $P$ and increases with $C$, and it diverges for $P=0$ or $C \rightarrow \infty$.

The thickness $h_{i}$ of the precursor film can be defined as the height at the lowest inflection point, so that its value is a solution of $\pi\left(h_{i}\right)=-P$ and is not dependent on $C$. Its value ranges from 1 for $P=0$ to $(n / m)^{1 /(n-m)}$ for $P=P_{v \text {, max }}$. Notice that the lower limit for $h_{i}$ implies that excess pressure $P$ must be positive, as expected for droplets [34]. The upper limit is also the maximum thickness of uniform stable films, so D1 solution can be interpreted as a sessile drop over a stable 
liquid film [12]. The length of the precursor film is increased when $C$ is reduced or when $P$ becomes larger.

The area of D1 solution varies not monotonically when $P$ or $C$ is modified. It increases as the values of $P$ and $C$ approach the boundaries of the region, diverging at the lower and the left boundaries. However, very near to the left boundary [curve $\left.\left(C_{v}, P_{v}\right)\right]$, the increase of the area is due to the enlargement of the film precursor (as can be seen in Fig. 6), but the bulk of the drop remains unchanged. On the contrary, very near to the lower boundary the area accumulates in the bulk of the drop while the film precursor remains finite.

When $C \rightarrow \infty$ the profile is parabolic with maximum thickness $C / P$, width $2 \sqrt{2 C} / P$, and cross-sectional area $2(2 C)^{3 / 2} /\left(3 P^{2}\right)$. This limit corresponds to the classical case of large droplets for which the effect of the disjoining-conjoining pressure is negligible and the curvature for the whole profile is $P$. More interesting is the limit $C \rightarrow C_{v}^{+}$. Figure 6(a) shows that for a given value of $P=P_{v}$, as $C$ decreases the shape in the bulk region of the droplet keeps constant and, notably, the length of the precursor film, $w_{p}$, increases following a $-a_{1} \log \left[a_{2}\left(C_{v}-C\right)\right]$ law, as shown in Fig. 6(b). The values of the positive constants $a_{1}$ and $a_{2}$ depends on $P$. Interestingly, solutions D3 and D1 with the same $P$ and $\left|C-C_{v}\right|$ share similar macroscopic profiles, and the period of D3 solutions for $C \rightarrow C_{v}$ also follows a logarithmic law.

In Ref. [17] Starov and Velarde studied static drops with infinite precursor film imposing only qualitative conditions for the disjoining pressure [the disjoining pressure given by Eq. (3) satisfies these conditions]. They found that a drop can be described as having three regions: a central circular cap, where capillary force dominates, a flat equilibrium film in front of the drop, and a transition zone between the two previous ones, where both capillary and disjoining-conjoining pressures are equally important. In this transition region, the profile tends asymptotically to the equilibrium thickness in a exponential way. It is expected that these results hold for D1 solution, with some modifications due to the finiteness of the precursor film. Its thickness was defined as the value of $h$ in the lowest inflection point, so to get a simple expression for the precursor film we solved Eq. (7) replacing $v(h)$ by its expansion up to second order around the inflection point, where $\pi(h)=-P$. From this, one obtains

$$
h=h_{i}+\sqrt{\frac{2 a}{b}} \sinh \left[\sqrt{b}\left(x_{i}-x\right)\right],
$$

where $x_{i}$ is the place where the inflection point is located, $h_{i}=h\left(x_{i}\right), a=v\left(h_{i}\right)-P h_{i}+C$, and $b=\frac{d^{2} h}{d h^{2}}\left(h_{i}\right)$. Notice that according to Eq. (13), $h$ decays exponentially to $h_{i}$, in the same way as was shown in Ref. [17], but near the extreme of the precursor film $h$ moves away exponentially. This last behavior was not observed in Ref. [17] because the authors considered only infinite precursor films. Besides, Eq. (13) also describes the profile around the highest inflection point. On the other, expanding $v(h)$ up to first order around the maximum height $h_{m}$ of the drop [defined by $v\left(h_{m}\right)=P h_{m}+C$ ], from Eq. (7) an approximated expression for the central region of the profile is obtained,

$$
h=h_{m}-\frac{1}{2}\left[\pi\left(h_{m}\right)+P\right] x^{2},
$$

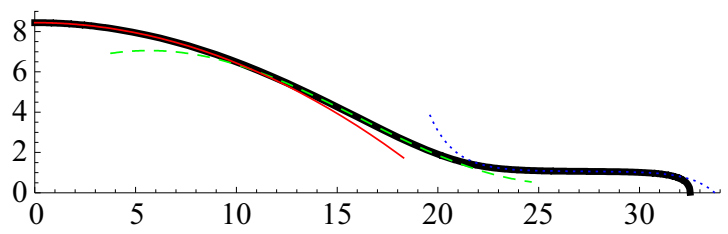

FIG. 7. Comparison of the solution D1 (thick black line) with the approximate solutions given by Eq. (14) describing the apex (thin red line), and by Eq. (13) describing the precursor film (thin dotted blue line) and the transition region between the previous ones (thin dashed green line).

where the expression inside the brackets is the opposite of the curvature at the apex. Notice that the disjoining pressure plays a role in the central region of the drop and, when $h_{m}$ is high enough to consider that $\pi\left(h_{m}\right) \approx 0$, only the surface tension is involved in agreement with Ref. [17]. In Fig. 7 we compare the solution D1 of Eq. (7) with the approximate solutions given by Eq. (13) for both inflection points and Eq. (14) for the central cap. It can be observed that with these three approximations almost the whole profile is successfully covered. Finally, note that Eqs. (13) and (14) do not depend on the specific expression adopted for $v(h)$ (as that used here), so that the results shown in this paragraph are valid for any $v(h)$ with the same qualitative shape as in Fig. 1.

\section{STATIC SOLUTIONS WITHOUT ASSUMING THE LUBRICATION APPROXIMATION}

The solutions presented in Sec. III were obtained assuming that $\alpha\left|h^{\prime}\right| \ll 1$, where $\alpha=h_{*} / x_{c}$, which means that the slope of the profile with its dimensions restored must be small. Thus, the dimensionless curvature $k$ was simplified to

$$
k=\frac{h^{\prime \prime}}{\left[1+\left(\alpha h^{\prime}\right)^{2}\right]^{3 / 2}} \approx h^{\prime \prime} .
$$

However, the condition $\alpha\left|h^{\prime}\right| \ll 1$ is not satisfied by the solutions D1, D2 (for $h \rightarrow 0$ ), ND1 and ND2 (for $h \rightarrow \infty$ ), and it may be not satisfied by solution D3. This fact justifies to analyze what happens with the static solutions when the complete expression for the curvature is considered. In this case, the Eq. (7) is modified to

$$
h^{\prime}= \pm \frac{1}{\alpha} \sqrt{\frac{1}{\left\{\alpha^{2}[v(h)-P h+C]-1\right\}^{2}}-1 .}
$$

Now, to ensure that $h$ is real it must be satisfied that

$$
P h-C+\frac{2}{\alpha^{2}} \geqslant v(h) \geqslant P h-C \text {. }
$$

Then, a graph of $v(h), P h-C$, and $P h-C+2 / \alpha^{2}$ versus $h$ is necessary to analyze the possible static profiles. Notice that now it is possible that the profile has points with infinite slope and nonvanishing $h$, and these points are the intersections of $v(h)$ with $P h-C+1 / \alpha^{2}$.

A strong novelty introduced in Eq. (16) compared to Eq. (7) is that there is no solution with $h=0$, as can be deduced from the behavior of $v(h)$ when $h \rightarrow 0$ and requiring nonnegativity of the radicand of Eq. (16). Therefore, solutions D1, D2, and ND2 are destroyed when the full expression of curvature is 

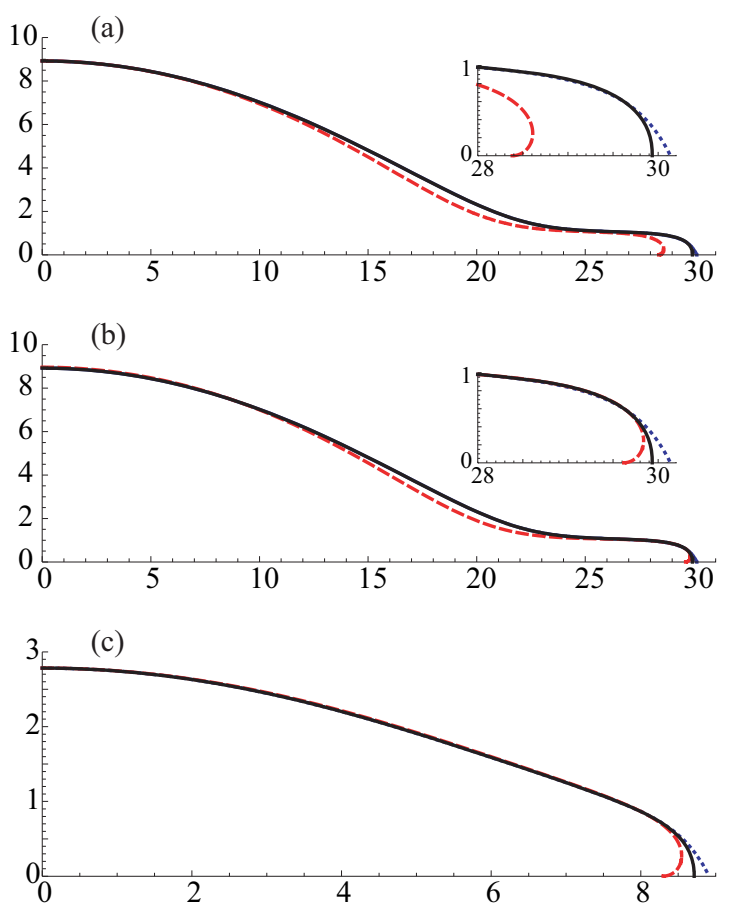

FIG. 8. The black full lines denote the D1 and D2 solutions obtained solving Eq. (7) given by the lubrication approximation, with the potential without cutoff. Complete curvature solutions numerically obtained from Eq. (16) with potential given by Eq. (18) are presented in red dashed lines. The blue dotted lines correspond to numerical solutions obtained by means of the lubrication approximation, Eq. (7), combined with the modified potential given by Eq. (18). In (a) the values of $P$ and $C$ for the three profiles are the same employed as that in Fig. 2. In (b) and (c) the values of $P$ and $C$ for the solution with full curvature were chosen to maximize the agreement between the profiles $(P=0.049745$ and $C=0.55135$ for D 1 and $P=0.15712$ and $C=0.732$ for D2). In all cases $n=3, m=2, \alpha=1$, and the values of $h_{c}$ were chosen to obtain $h^{\prime}=0$ for $h=0$ when the full curvature is considered.

considered. However, this is an artifact due to the nonphysical divergent behavior of $v$ for $h \rightarrow 0$ ( $v$ still is singular at $h=0$ when the lubrication approximation is used, but the radicand of Eq. (7) is positive when $h \rightarrow 0$, so no problem arises). To avoid this problem we introduce a cutoff, so that $\Pi(h)$ is changed slightly only about $h=0$ to eliminate the divergence but without changes for larger $h$. One (arbitrary) way to do this is introducing a prefactor in the expression for $\pi(h)$ as it was done in Ref. [40]:

$$
\pi(h)=\left[1-e^{-\left(h / h_{c}\right)^{n}}\right]\left(\frac{1}{h^{n}}-\frac{1}{h^{m}}\right),
$$

with $h_{c}>0$. Figure 8 compares the solutions D1 and D2 obtained considering the full expression of curvature $k$ with the molecular interaction modeled by Eq. (18) with those shown in Fig. 2. We also show, in dotted blue lines, the profiles obtained by means of the lubrication approximation, Eq. (7), combined with the modified potential given by Eq. (18) to appreciate to what extent the differences come from using the complete expression for the curvature and to what extent from introducing the cutoff in the disjoining-conjoining pressure.

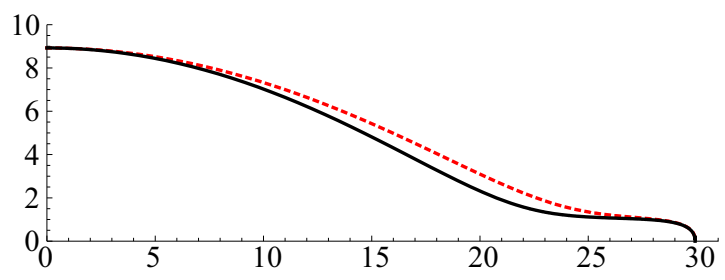

FIG. 9. Comparison of the solution D1 for the 2D case (black full line) with the corresponding 3D solution (red dashed line). For both solutions $n=3$ and $m=2$, and $P$ and $C$ as in Fig. 2 for the plane symmetry, and $P=0.07617$ for the axial symmetry, which was chosen so that both solutions have the same radius.

As expected, the lubrication solutions shown in Fig. 2 are only slightly modified in the region close to $h=0$ when the modified potential $\pi$ is employed. In Fig. 8(a) both profiles were obtained with the same values of $P$ and $C$ as in Fig. 2. Although there is an acceptable agreement between these profiles, the differences can be even smaller choosing adequately the values of $P$ and $C$ for the solution with full curvature as is shown in Fig. 8(b). In Fig. 8(c) we compare the solution D2.

From Eqs. (15) and (16) it is clear that the solutions presented here tend to the solutions with complete expression of curvature as $\alpha$ becomes smaller. In Fig. 8, the red dashed line has $\alpha=1$, and if this value were smaller the profile would be more like the profile obtained from the lubrication approximation (the black full line). Besides, for Figs. 8(a) and 8 (b) the maximum slope (in the bulk of the drop, omitting the precursor film) is around 0.517 , which implies that the contact angle is $27^{\circ}$. It can be observed that there are minor differences between the profile obtained by means of the lubrication approximation with that obtained using the complete expression of curvature, even for a contact angle close to $30^{\circ}$ (agreeing with Ref. [41]). This reinforces the idea that the lubrication approximation gives confident results even in cases where the contact angle is not close to zero.

\section{COMPARISON BETWEEN 2D AND AXISYMMETRIC 3D STATIC SOLUTIONS}

Up to here we have assumed plane symmetry. Now, we shall change the symmetry and we look for axisymmetric solutions and compare them with the $2 \mathrm{D}$ solutions. In this case, assuming again that $\alpha\left|h^{\prime}\right| \ll 1$ the curvature can be approximated,

$$
k=\frac{h^{\prime}}{x\left[1+\left(\alpha h^{\prime}\right)^{2}\right]^{1 / 2}}+\frac{h^{\prime \prime}}{\left[1+\left(\alpha h^{\prime}\right)^{2}\right]^{3 / 2}} \approx \frac{1}{x} h^{\prime}+h^{\prime \prime},
$$

where $x$ is now the radial coordinate. The equation for $h$ is

$$
\frac{1}{x}\left(x h^{\prime}\right)^{\prime}+\pi=-P .
$$

Figure 9 compares the planar symmetry solution D1 with the corresponding axial-symmetry one. The parameters of these solutions were chosen so that they have the same height and width. It can be observed that the solutions are quite similar. The same was found for the solution D2. The periodic solution D3 in the axially symmetric case becomes an oscillating solution whose amplitude decreases as $x$ is larger. 


\section{DISCUSSION AND CONCLUSIONS}

We have presented the complete family of solutions describing the static free surface of a fluid that rests on a solid flat substrate with which interacts through the potential Eq. (3). This potential includes a repulsive and an attractive term, and it is characterized by the exponents $n$ and $m$. There are five nontrivial solutions for all $n$ and $m$. Three of them can be described as dropletlike solutions, which we called D1, D2, and D3, and the other two, ND1 and ND2, are divergent. For the widely employed potential with $n=3$ and $m=2$ [5,8], we present a closed form solution, Eq. (10), which comprises the five solutions. The most interesting solution is D1, because it describes a finite volume droplet with a finite-length precursor film. Varying the integration constants $P$ and $C$ it is possible to vary the extension of the precursor film, the width, the height, and the volume of the droplet. To the best of our knowledge, this is the first time that the analytical solution for droplets with finite-length precursor films is reported. A similar family of solutions was numerically obtained in Ref. [42], but for a disjoining pressure, which includes a dependence on the slope of the profile and with $n=9$ and $m=3$.

Equation (7) and its five static solutions were obtained with two assumptions: plane symmetry and small slope, what it allows to approach the curvature by $h^{\prime \prime}$. Here we analyzed the effects of these assumptions and the limits that they impose. Plane symmetry is a quite strong condition for real situations, so that strict applicability of the solutions presented here is limited to few situations. For example, they may be useful to describe the transversal profile of a long straight rivulet flowing steadily down an inclined surface $[41,43]$, or to describe a drop of liquid sandwiched between two vertical plates separated by a very narrow gap [44]. However, the comparison shown in Fig. 9 between a 2D solution with the corresponding 3D solution with axial symmetry shows that the relevance of these $2 \mathrm{D}$ solutions mainly lies on the possibility of that they can be used to describe the transverse cross section of the axisymmetric shapes.

We compare solutions D1 and D2 with the equivalent ones obtained using the full expression of curvature. The main conclusion is that there are tiny differences between them which are not sufficient to invalidate the results obtained with the lubrication approach. Thus, the lubrication approximation is also useful to describe situations where the contact angle is even as large as $30^{\circ}$.

To employ the full expression of the curvature and obtain solutions with $h=0$, it is necessary to avoid the nonphysical divergence at $h=0$, and this can be done by introducing a cutoff in the disjoining-conjoining pressure $\pi(h)$, i.e., a parameter $h_{c}$ such that for $h \lesssim h_{c}$ the disjoining-conjoining pressure saturates and tends to a finite value [34]. In this way the solutions are not modified in the bulk of the droplet and only the details of the profile for $h \lesssim h_{c}$ depend on the way the cutoff is introduced. The exact form of $\pi$ for $h \lesssim h_{c}$ has a negligible effect on the profile, so one just make a choice which makes the algebra simpler. To do this, we arbitrarily adopt the expression given in Eq. (18) just to be able to calculate the profiles with full curvature (other ways to introduce a cutoff can be seen in Refs. [45-47]). At this point it is convenient to mention that disjoining-pressure may be obtained from molecular dynamic simulations [39] and from density functional theory [48].

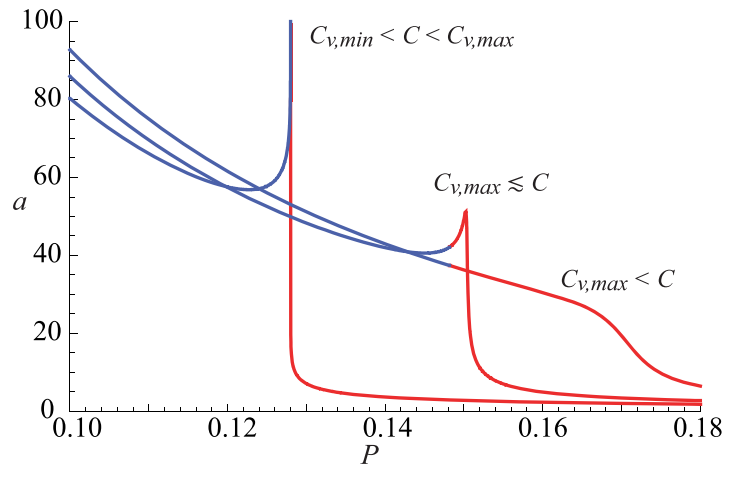

FIG. 10. Dimensionless area $a$ as function of $P$ for three fixed values of $C(0.64,0.67$, and 0.7$)$. The dark (blue) part of each curve is for $\mathrm{D} 1$ solution and the gray (red) one is for $\mathrm{D} 2$.

In a real situation, the volume of liquid deposited on a substrate is obviously always finite and, in consequence, the static shape that the surface finally reached after a transient period will be described by the solution D1 or D2 (if the limits of the substrate are not reached by the liquid). Then, a question naturally emerges: given a pair liquid-solid substrate, which implies according to what is shown toward the end of Sec. III a unique given value of $C$, and a given value of the area (volume in the three dimensional case) of the liquid, what shape will the drop adopt, D1 or D2? We can give some partial answers to this question. When $C<C_{v \text {, min }}$ only D2 drops are possible, independently of the amount of liquid. To see what happens when $C>C_{v \text {,min }}$ we show in Fig. 10 the dimensionless area $a=\int h d x$ as function of $P$ with constant $C$, for three values of $C$ (for this figure we use $n=3$ and $m=2$ because in this case a closed expression for $a$ can be obtained; see the Appendix). It can be observed that if $C_{v \text {, min }}<C<C_{v \text {, max }}$, there is a local minimum of the area $a_{m}$ so that for an area lesser than $a_{m}$ only a D2 solution is possible, and for a bigger area two D1 and one D2 solution exist. For $C$ bigger than $C_{v \text {,max }}$ but very close to it, the curve has also a local maximum $a_{M}$ such as if $a>a_{M}$ only a solution of the kind D1 can be attained, and for $a_{m}<a<a_{M}$ one D1 and two D2 solutions are possible. For bigger values of $C$ the curve decreases monotonically and for large or small areas only D1 or D2 solutions are possible, respectively. So, for those values of $C$ and $a$ for which there is only one possible solution, this solution will describe the final shape of the drop, but in situations with more than one possible solution the selected final state depends on the stability and on the energy of those solutions, an issue out of the scope of the present work.

Finally, the previous paragraph may induce to think that the solutions D3, ND1, and ND2 are irrelevant because they have infinite area. However, they may be relevant to describe the final shape of the free surface of a finite amount of a liquid inside a recipient with a finite extension (this situation was analyzed in Ref. [12] in the simplest case when the contact angle with the lateral walls is $90^{\circ}$ ).

\section{ACKNOWLEDGMENTS}

C.A.P., J.R.M.I., and J.M.G. acknowledge Grants PIP No. 299 and PIP No. 356 from Consejo Nacional de Investiga- 
ciones Científicas y Técnicas, and Grant PICT No. 1707 from Agencia Nacional de Promoción Científica y Técnica.

\section{APPENDIX: THE AREA AND HALF-WIDTH OF D1 AND D2 FOR $n=3$ AND $m=2$}

Here we show the derivation and the final expression of the dimensionless area $a$ for the solutions D1 and D2 when $n=3$ and $m=2$, defined as

$$
a=2 \int_{0}^{x_{\mathrm{end}}} h d x .
$$

We have assumed that the solutions are symmetric around $x=0$, and $x_{\text {end }}$ is the place where $h=0$.

In the case $n=3$ and $m=2$, Eq. (7) can be written as (only the decreasing half is taken)

$$
\begin{aligned}
h^{\prime} & =-\sqrt{2} \sqrt{\frac{1}{2 h^{2}}-\frac{1}{h}-P h+C} \\
& =-\frac{1}{h} \sqrt{-2 P\left(h-h_{1}\right)\left(h-h_{2}\right)\left(h-h_{3}\right)},
\end{aligned}
$$

where $h_{1}, h_{2}$, and $h_{3}$ are the roots of $-2 P h^{3}+2 C h^{2}-2 h+1$. In the case of solutions D1 and D2 this polynomial has only one real root, which is the maximum height of the solution, and in the following we assume is $h_{1}$. By means of a change of variable and using Eq. (A2), Eq. (A1) can be written as

$$
\begin{aligned}
a & =2 \int_{h_{1}}^{0} \frac{h}{h^{\prime}} d h \\
& =-2 \int_{h_{1}}^{0} \frac{h^{2}}{\sqrt{-2 P\left(h-h_{1}\right)\left(h-h_{2}\right)\left(h-h_{3}\right)}} d h .
\end{aligned}
$$

The last integral can be calculated, and after some algebra the expression for the dimensionless area is

$$
\begin{aligned}
a= & \frac{2}{3 P}\left\{1+2 C x_{\text {end }}\right. \\
& +\sqrt{\left.\frac{2}{P\left(h_{3}-h_{1}\right)}\left[\mathrm{F}\left(\left.\psi\right|_{h=0}, \tau\right)-\mathrm{F}\left(\left.\psi\right|_{h=h_{1}}, \tau\right)\right]\right\},}
\end{aligned}
$$

where $\psi$ and $\tau$ were defined in Eq. (10), and $x_{\text {end }}$ is the halfwidth of the solution D1 or D2:

$$
\begin{aligned}
x_{\text {end }}= & \sqrt{\frac{2}{P\left(h_{3}-h_{1}\right)}}\left\{\left(h_{1}-h_{3}\right)\left[\mathrm{E}\left(\left.\psi\right|_{h=0}, \tau\right)-\mathrm{E}\left(\left.\psi\right|_{h=h_{1}}, \tau\right)\right]\right. \\
& \left.-h_{1}\left[\mathrm{~F}\left(\left.\psi\right|_{h=0}, \tau\right)-\mathrm{F}\left(\left.\psi\right|_{h=h_{1}}, \tau\right)\right]\right\} .
\end{aligned}
$$

[1] S. F. Kistler and P. M. Schweizer, Liquid Film Coating: Scientific Principles and Their Technological Implications (Chapman \& Hall, New York, 1997).

[2] S. J. Weinstein and K. J. Ruschak, Annu. Rev. Fluid Mech. 36, 29 (2004).

[3] J. B. Grotberg, Phys. Fluids 23, 021301 (2011).

[4] R. J. Braun, Annu. Rev. Fluid Mech. 44, 267 (2012).

[5] L. W. Schwartz, R. V. Roy, R. R. Eley, and S. Petrash, J. Colloid Interface Sci. 234, 363 (2001).

[6] A. Sharma and R. Verma, Langmuir 20, 10337 (2004).

[7] P. G. de Gennes, Rev. Mod. Phys. 57, 827 (1985).

[8] A. Oron, S. H. Davis, and S. G. Bankoff, Rev. Mod. Phys. 69, 931 (1997).

[9] D. Bonn, J. Eggers, J. Indekeu, J. Meunier, and E. Rolley, Rev. Mod. Phys. 81, 739 (2009).

[10] R. V. Craster and O. K. Matar, Rev. Mod. Phys. 81, 1131 (2009).

[11] F. Dörfler, M. Rauscher, and S. Dietrich, Phys. Rev. E 88, 012402 (2013).

[12] C. A. Perazzo, J. R. Mac Intyre, and J. M. Gomba, Phys. Rev. E 89, 043010 (2014).

[13] A. Sharma, Langmuir 9, 3580 (1993).

[14] V. M. Starov, Adv. Coll. Interface Sci. 39, 147 (1992).

[15] V. Mitlin, J. Colloid Interface Sci. 227, 371 (2000).

[16] A. L. Bertozzi, G. Grün, and T. P. Witelski, Nonlinearity 14, 1569 (2001).

[17] V. M. Starov and M. G. Velarde, J. Phys.: Condens. Matter 21, 464121 (2009).

[18] U. Thiele, M. G. Velarde, K. Neuffer, and Y. Pomeau, Phys. Rev. E 64, 031602 (2001).

[19] J. Diez, A. González, J. Gomba, R. Gratton, and L. Kondic, Physica D: Nonlin. Phenom. 209, 49 (2005).
[20] J. M. Gomba, J. Diez, A. G. González, and R. Gratton, Phys. Rev. E 71, 016304 (2005).

[21] J. M. Gomba and C. A. Perazzo, Phys. Rev. E 86, 056310 (2012).

[22] B. V. Derjaguin and M. M. Kusakov, Proc. Acad. Sci. USSR Chem. 5, 741 (1936).

[23] N. V. Churaev and V. D. Sobolev, Adv. Coll. Interface Sci. 61, 1 (1995).

[24] L. W. Schwartz and R. R. Eley, J. Colloid Interface Sci. 202, 173 (1998).

[25] V. S. Mitlin, Coll. Surf. A 89, 97 (1994).

[26] G. F. Teletzke, H. T. Davis, and L. E. Scriven, Chem. Eng. Comm. 55, 41 (1987).

[27] L. W. Schwartz and R. V. Roy, Phys. Fluid 16, 569 (2004).

[28] J. M. Gomba and G. M. Homsy, J. Fluid Mech. 647, 125 (2010).

[29] J. M. Gomba and G. M. Homsy, Langmuir 25, 5684 (2009).

[30] V. S. Mitlin, J. Colloid Interface Sci. 156, 491 (1993).

[31] L. Leger and J. F. Joanny, Rep. Prog. Phys. 55, 431 (1992).

[32] H. Hervet and P.-G. De Gennes, C. R. Acad. Sci. Paris 299, 499 (1984).

[33] G. Kitavtsev, L. Recke, and B. Wagner, Nonlinearity 24, 2347 (2011).

[34] V. Starov, M. Velarde, and C. Radke, Wetting and spreading dynamics, Surfactant science series (CRC Press, Boca Raton, 2007).

[35] J. R. Mac Intyre, J. M. Gomba, and C. A. Perazzo, J. Eng. Math. 101, 55 (2016).

[36] J. Eggers, Phys. Rev. E 72, 061605 (2005).

[37] H. E. Huppert, J. Fluid Mech. 121, 43 (1982).

[38] J. Gratton and F. Minotti, J. Fluid Mech. 210, 155 (1990).

[39] N. Tretyakov, M. Müller, D. Todorova, and U. Thiele, J. Chem. Phys. 138, 064905 (2013). 
[40] A. L. Bertozzi and M. Pugh, Nonlinearity 7, 1535 (1994).

[41] C. A. Perazzo and J. Gratton, J. Fluid Mech. 507, 367 (2004).

[42] T. Yi and H. Wong, J. Colloid Interface Sci. 313, 579 (2007).

[43] A. J. Tanasijczuk, C. A. Perazzo, and J. Gratton, Eur. J. Mech. B 29, 465 (2010).

[44] E. Pitts, J. Fluid Mech. 59, 753 (1973).
[45] Y. Solomentsev and L. R. White, J. Colloid Interface Sci. 218, 122 (1999).

[46] G. O. Berim and E. Ruckenstein, J. Phys. Chem. B 108, 19330 (2004).

[47] M. E. Diaz, J. Fuentes, R. L. Cerro, and M. D. Savage, J. Colloid Interface Sci. 343, 574 (2010).

[48] A. P. Hughes, U. Thiele, and A. J. Archer, J. Chem. Phys. 142, 074702 (2015). 